\title{
Experimental study on energy absorption characteristics of motorcycle front wheel- tyre assembly in frontal impact
}

\begin{abstract}
This article presents a study on the frontal impact properties of the motorcycle front wheelठ tyre assembly using squared change of velocity and dissipated impact energy as response variables. The laboratory-scaled impact tests were performed on the wheelठ tyre assemblies using pendulum impact test apparatus according to a $25 \overline{\mathrm{I}} 1 \mathrm{~V}$ fractional factorial design with four replicates. Five parameters that are included in the experiment were impact speed $(\mathrm{S})$, impact mass (M), tyre inflation pressure level (P), contact geometry of striker (G) and offset distance of impact location from axle (D). The test specimen used in the study was a spoked wheel of size $1.40 \times 17$. Minitab has been employed to support the entire experimental process. The collected experimental data were organized, and factorial analysis was performed. The significant factors influencing the impact responses of the wheelठ tyre assembly were identified, which are S, M, P, D, SM and SD. The associated empirical models were then established and presented, followed by the factorial plots for the respective significant factors. The curves demonstrating the impact response of the wheelठ tyre assembly under various impact conditions were generated from the developed models to illustrate the impact response of the wheelठ tyre assembly under various impact conditions within the experimental region. Comparison was made to the values of dissipated energy predicted by the developed model and the values of impact energy from fundamental kinetic energy equation, and it was found that the model is consistent with the physical condition within the experimental region.
\end{abstract}

Keyword: Design of experiments; Impact energy absorption; Motorcycle crashworthiness; Wheel-tyre assembly 